\title{
Benefits of resection for metachronous lung cancer
}

\author{
Richard J. Battafarano, MD, PhD \\ Seth D. Force, MD \\ Bryan F. Meyers, MD \\ Jennifer Bell, BSN \\ Tracey J. Guthrie, BSN \\ Joel D. Cooper, MD \\ G. Alexander Patterson, MD
}

See related editorial on page 633.
From the Department of Surgery, Division of Cardiothoracic Surgery, Washington University School of Medicine, St Louis, Mo.

Read at the Eighty-third Annual Meeting of The American Association for Thoracic Surgery, Boston, Mass, May 4-7, 2003.

Received for publication May 2, 2003; revisions received Aug 4, 2003; accepted for publication Aug 11, 2003.

Address for reprints: Richard J. Battafarano, $\mathrm{MD}$, $\mathrm{PhD}$, One Barnes-Jewish Plaza, 3107 Queeny Tower, St Louis, MO 63110-1013 (E-mail: battafarano@msnotes. wustl.edu).

J Thorac Cardiovasc Surg 2004;127:836-42 0022-5223/\$30.00

Copyright (C) 2004 by The American Association for Thoracic Surgery

doi:10.1016/j.jtcvs.2003.08.055
Objectives: The benefits of resection for metachronous lung cancer are not well described. The objective of this study was to evaluate the safety and efficacy of surgical resection for metachronous lung cancers.

Methods: We reviewed the charts of all patients who underwent a second resection for a metachronous lung cancer from July 1, 1988, to December 31, 2002. Type of resection, operative morbidity, mortality, and survival by stage were analyzed. Survival was determined by using the Kaplan-Meier survival method. All patients were pathologically staged by using the 1997 American Joint Committee on Cancer standards.

Results: Pulmonary resections were performed in 69 patients who had undergone a previous resection. The mean interval between the first and second resection was 2.4 \pm 2.5 years. Seventy-three percent of patients presented with stage I cancers, 9\% with stage II cancers, and $17 \%$ with stage III cancers. Lobectomy and wedge resection were performed with equal frequency ( $42 \%$ each) for the metachronous cancers. Operative mortality for the second resection was 5.8\%. The mean follow-up after the second resection was 37 months. Overall 5-year actuarial survival for the entire group after the second resection was $33.4 \%$.

Conclusions: Operations for metachronous cancers provided survival that approximated the expected survival for lung cancer. Surgical intervention should be considered as a safe and effective treatment for resectable metachronous lung cancer in patients with adequate physiologic pulmonary reserve.

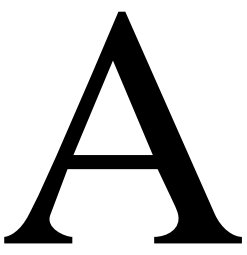

pproximately $50 \%$ of the 170,000 patients with newly diagnosed lung cancer will have localized disease that is amenable to potentially curative therapy. ${ }^{1,2}$ Patients with stage I and stage II non-small cell lung cancers (NSCLCs) who undergo complete surgical resection have a favorable prognosis, with actuarial 5 -year survivals that range from $55 \%$ to $75 \%$ and $35 \%$ to $45 \%$, respectively. ${ }^{1,3-5}$ Factors that limit long-term survival include the patients' underlying comorbidity, ${ }^{6}$ tumor recurrence, and the development of second primary lung cancers. ${ }^{7-9}$ Although there has been considerable debate concerning the usefulness of postoperative surveillance in decreasing the mortality associated with recurrent NSCLC, ${ }^{10-14}$ early identification and surgical resection of second primary lung cancers has been associated with encouraging results. ${ }^{15-22}$

The risk of development of a new primary lung cancer after undergoing definitive surgical therapy for an NSCLC is estimated to be $1 \%$ to $2 \%$ per patient per year. ${ }^{8,9}$ However, differentiating synchronous primary lung cancers from intrapulmonary metastases and metachronous lung cancers from recurrent lung cancer can be difficult when the tumor histology is the same. According to the 
guidelines proposed by Martini and Melamed, ${ }^{23}$ tumors with the same histology might be considered synchronous if they are found in a different segment, lobe, or lung; there is no carcinoma in lymphatics common to both; and there is no evidence of extrapulmonary metastasis at the time of diagnosis. Tumors with the same histology might be considered metachronous if there has been a disease-free interval between cancers of at least 2 years or the second tumor is found in a different segment, lobe, or lung; there is no carcinoma in the lymphatics common to both; and there is no evidence of extrapulmonary metastasis at the time of diagnosis. Although these guidelines remain useful, the routine use of spiral computed tomographic (CT) imaging in the preoperative staging and postoperative surveillance of patients with NSCLCs has been responsible for the identification of many additional small cancers.

Because accurate differentiation of synchronous or metachronous tumors from intrapulmonary metastases or recurrent lung cancer is not always clinically possible, patients with adequate pulmonary and physiologic reserve have undergone surgical resection of these second lesions. The purpose of this study was to determine the effect of completely resected metachronous NSCLC on survival.

\section{Methods}

\section{Population Under Study}

A database analysis of our cardiothoracic surgery tumor registry was performed. From July 1, 1988, to December 31, 2002, a total of 2107 patients underwent pulmonary resection for NSCLC. During this period, 69 patients underwent multiple resections for NSCLC. The surgical pathology reports of all patients who underwent multiple resections identified by this database query were individually reviewed, and the final pathologic staging was assigned by using the 1997 revisions in the International System for Staging Lung Cancer. Patients who underwent anatomic resection of an NSCLC who had previously undergone excision by means of wedge excision in the same lobe were excluded from this analysis.

Tumor recurrence, patient survival, and cause of death were determined for each patient. Follow-up information on all patients was acquired within the last 6 months through clinic follow-up notes, direct patient or family contact, contact with the patient's primary care physician, and review of all death certificates. This study represents a secondary data analysis of a prospective cohort study. Approval for this study was granted by the Washington University School of Medicine Human Studies Committee.

\section{Statistical Analysis}

Descriptive statistics were used to describe the patients' characteristics and outcomes. Normally distributed continuous data are expressed as means \pm SD throughout. Medians with ranges are used when continuous data are not normally distributed. Categoric data are expressed as counts and proportions. Fisher exact or $\chi^{2}$ tests were used to analyze the categoric data.
TABLE 1. Pathologic stage

\begin{tabular}{lcc}
\hline & $\begin{array}{c}\text { First resection } \\
\text { (n = 69) }\end{array}$ & $\begin{array}{c}\text { Second resection } \\
\text { (n = 69) }\end{array}$ \\
\hline Stage IA & $30(43.5 \%)$ & $34(49.3 \%)$ \\
Stage IB & $14(20.3 \%)$ & $16(23.2 \%)$ \\
Stage IIA & $5(7.2 \%)$ & $3(4.3 \%)$ \\
Stage IIB & $11(15.9 \%)$ & $3(4.3 \%)$ \\
Stage IIIA & $5(7.2 \%)$ & $4(5.8 \%)$ \\
Stage IIIB & $3(4.3 \%)$ & $8(11.6 \%)$ \\
Stage IV & $1(1.4 \%)$ & $1(1.4 \%)$ \\
\hline
\end{tabular}

Kaplan-Meier (product-limit) graphs were used to demonstrate survival over time and freedom from recurrence of disease. Survival comparison between groups of patients was completed by using the Mantel-Haenszel log rank test.

All data analysis was performed with Systat software (Systat 10.0 for Windows; SPSS Inc, Chicago, Ill).

\section{Results}

\section{Patient Demographics}

Of the 69 patients in this experience, $36(52 \%)$ were male and $33(48 \%)$ were female. The mean ages at the time of the first and second resections were $65.1 \pm 8.7$ and $67.5 \pm 8.9$ years, respectively. The majority of patients had stage I disease at the time of both the first (44/69 [64\%]) and second (50/69 [73\%]) resections (Table 1). However, the remaining 25 patients were found to have more advanced disease at the time of the first resection because of lymph node metastases $(n=16)$, chest wall invasion $(n=5)$, or multifocal lung cancer $(n=4)$. At the time of the second resection, 19 patients were found to have more advancedstage disease because of lymph node metastases $(n=7)$, chest wall invasion $(\mathrm{n}=3)$, or multifocal lung cancer $(\mathrm{n}=$ 9).

Pulmonary function test results were available for review on 43 of 69 patients before the initial resection and on 45 of 69 patients before the second resection (Table 2). The mean forced expiratory volume in 1 second (percent predicted) before the first and second resections for this group of patients was $81 \%$ and $65 \%$, respectively. The mean arterial blood gas $\mathrm{PaO}_{2}$ and $\mathrm{PaCO}_{2}$ values were also in the normal range for this group before the first and second resections.

Sixty-one $(88 \%)$ patients underwent anatomic resection of their lung cancer at the time of the first resection (Table 3 ). The majority underwent lobectomy (78\%). However, 6 (9\%) patients required a more extensive operation to ensure complete resection. Nine patients with small lesions underwent limited pulmonary resection by means of segmentectomy $(1 \%)$ or wedge excision $(12 \%)$. Surgical resection of the metachronous NSCLC was accomplished with anatomic resection in $40(58 \%)$ patients. Twenty-nine (42\%) patients underwent lobectomy, 2 (3\%) patients underwent bilobectomy, and 4 (6\%) patients underwent completion pneumo- 
TABLE 2. Preoperative pulmonary function test results

\begin{tabular}{|c|c|c|}
\hline & $\begin{array}{l}\text { First resection } \\
\qquad(n=43)\end{array}$ & $\begin{array}{l}\text { Second resection } \\
\quad(n=45)\end{array}$ \\
\hline $\mathrm{FEV}_{1}(\%$ predicted $)$ & $81.1 \pm 23$ & $65.2 \pm 17$ \\
\hline FVC (\% predicted) & $96.2 \pm 23$ & $86.4 \pm 19$ \\
\hline $\mathrm{Po}_{2}(\mathrm{~mm} \mathrm{Hg})$ & $78 \pm 10$ & $80 \pm 9$ \\
\hline $\mathrm{PcO}_{2}(\mathrm{~mm} \mathrm{Hg})$ & $39 \pm 8$ & $37 \pm 4$ \\
\hline
\end{tabular}

$F E V_{1}$, Forced expiratory volume in 1 second; $F V C$, forced vital capacity.

TABLE 3. Type of surgical resection performed

\begin{tabular}{lcc}
\hline & $\begin{array}{c}\text { First resection } \\
\text { (n = 69) }\end{array}$ & $\begin{array}{c}\text { Second resection } \\
\text { (n = 69) }\end{array}$ \\
\hline Lobectomy & $54(78.3 \%)$ & $29(42.0 \%)$ \\
Bilobectomy & $3(4.3 \%)$ & $2(2.9 \%)$ \\
Segmentectomy & $1(1.4 \%)$ & $5(7.2 \%)$ \\
Pneumonectomy & $3(4.3 \%)$ & $4(5.9 \%)$ \\
Wedge(s) & $8(11.7 \%)$ & $29(42.0 \%)$ \\
\hline
\end{tabular}

nectomy. Thirty-four (49\%) patients with small peripheral lesions underwent limited pulmonary resection by means of segmentectomy (7\%) or wedge excision (42\%) to preserve pulmonary function.

The metachronous lung cancer was in the contralateral lung in $52(75 \%)$ patients and the ipsilateral lung in 17 $(25 \%)$ patients. Forty-three (62\%) patients had tumors with the same histology, and 26 (38\%) had tumors with different histology (Table 4). Adenocarcinoma was the most frequently identified histologic subtype encountered: 40 (58\%) patients had a least one tumor that was an adenocarcinoma. Only 3 patients had bronchioloalveolar cancer identified in both lesions, and 3 patients had bronchioloalveolar cancer in addition to adenocarcinoma $(\mathrm{n}=1)$ or squamous cell cancer $(n=2)$. Forty $(58 \%)$ patients underwent resection of their metachronous lung cancer within 2 years of the initial resection.

\section{Survival}

Survival data were collected on each patient from the dates of the operation. There were $4(5.8 \%)$ postoperative deaths after resection of the metachronous NSCLC in this series. All 4 deaths were caused by respiratory failure associated with pneumonia. The 5-year actuarial survival for all patients after resection of metachronous NSCLC was 60.9\% and $33.4 \%$ after resection of the primary and metachronous lesions, respectively. The 5-year actuarial survival of patients who had stage I NSCLC at the time of the first resection was not significantly different than that of patients who had more advanced tumors initially resected $(73 \%$ vs $39 \%, P=.07$; Figure 1). However, the 5-year actuarial survival of patients who had metachronous stage I NSCLC resected was significantly better than that in patients with
TABLE 4. Histology of resected lesion from 69 patients

\begin{tabular}{lr}
\hline Same histology & 43 \\
Adenocarcinoma & 20 \\
Squamous & 18 \\
Sarcomatoid & 1 \\
Neuroendocrine & 1 \\
BAC & 3 \\
Different histology & 26 \\
Adenocarcinoma + squamous & 14 \\
Adenocarcinoma + mixed & 5 \\
Adenocarcinoma + neuroendocrine & 1 \\
Squamous + other & 2 \\
Adenocarcinoma + BAC & 2 \\
Squamous + BAC & 1 \\
Neuroendocrine + other & 1 \\
\hline
\end{tabular}

$B A C$, Bronchioloalveolar cancer.

more advanced metachronous lesions (42\% vs $10 \%, P=$ .01 ; Figure 2).

\section{Effect of Tumor Histology and Interval Between Resections on Survival in Patients With Metachronous NSCLC}

To determine whether specific subgroups of metachronous NSCLC had a more favorable prognosis, we examined survival in patients with multiple tumors with the same histology (which would include patients with intrapulmonary metastases) and those with different histology. There was no difference in survival between these groups (Figure 3 ). Because many investigators exclude patients with a short disease-free interval between resection of metachronous lesions, we examined survival in patients in whom the interval between resection of their metachronous lung cancers was less than 24 months and in those in whom the interval was greater than 24 months. There was no difference in survival between these groups (Figure 4).

\section{Discussion}

Patients successfully treated for their initial NSCLC have a risk of having a second NSCLC of approximately $1 \%$ to $2 \%$ per patient per year. Optimal management of metachronous lung cancer is affected by a number of factors, including the patient's pulmonary reserve, associated medical comorbidity, and the clinical stage of the second lung cancer. The 69 patients in this series who underwent resection of their metachronous lung cancer represented approximately $3 \%$ of all patients who underwent surgical intervention for NSCLC during this 14-year period at our institution and is similar to the incidence of metachronous lung cancer reported by other investigators. ${ }^{16,19,20}$ All of these series (including our own) likely underestimate the true incidence of metachronous lung cancer because only patients with the physiologic reserve to undergo a second resection are included.

Patients who underwent resection of metachronous lung cancer had overall 5-year survivals of $61 \%$ and $33 \%$ after 


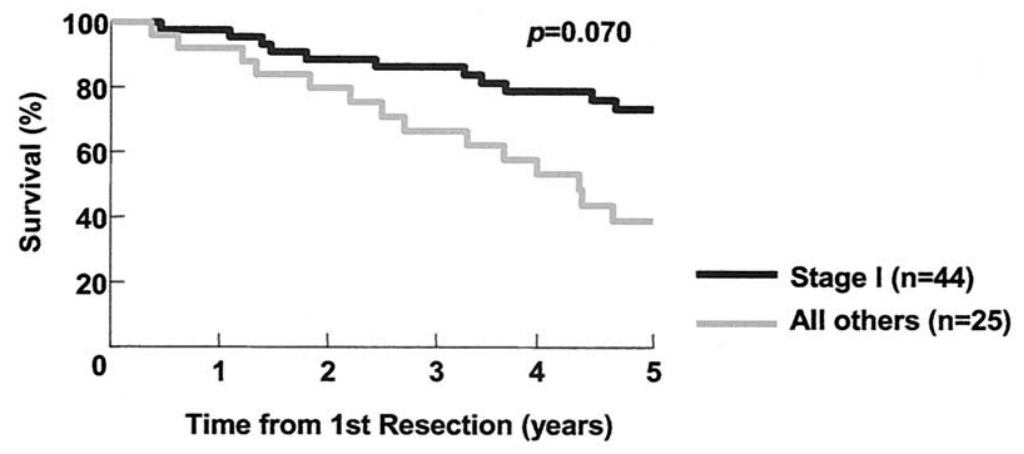

Figure 1. Five-year actuarial survival from the time of the first resection of the entire group stratified by the stage of the primary NSCLC.

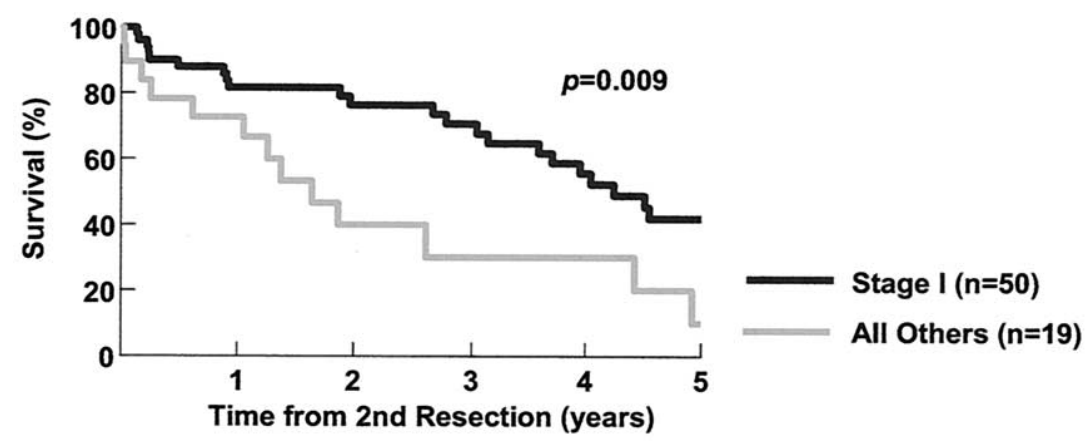

Figure 2. Five-year actuarial survival from the time of the second resection of the entire group stratified by the stage of the metachronous NSCLC.

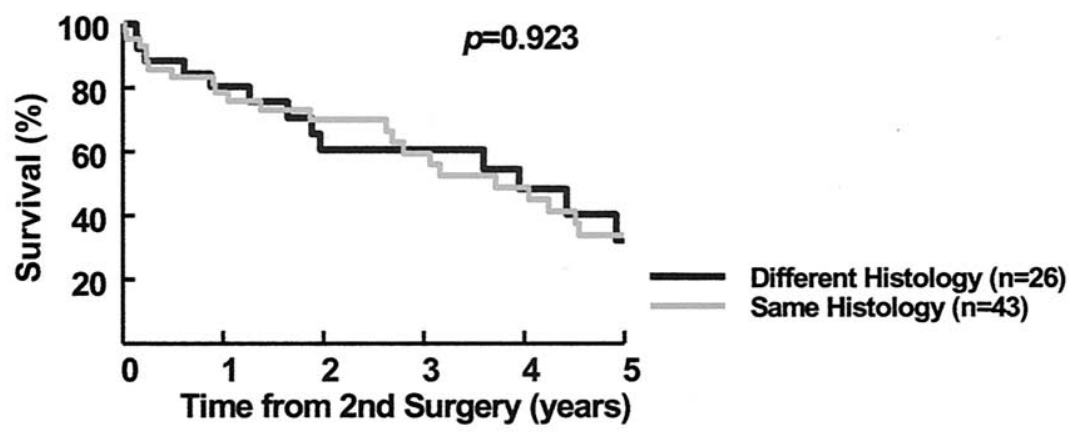

Figure 3. Five-year actuarial survival from the time of the second resection of the entire group stratified by tumor histology.

resection of their initial and metachronous lung cancers, respectively. There are many factors that likely contribute to the favorable survival observed in patients who underwent resection of their metachronous lung cancers in this analysis. Patients selected for resection had adequate physiologic reserve, as evidenced by a relatively low operative mortality (5.8\%). The final pathologic stage of the metachronous tumor was stage I in $73 \%$ of the patients. Finally, the majority (58\%) of these patients underwent anatomic resec- tion of their second NSCLC by means of segmentectomy, lobectomy, bilobectomy, or completion pneumonectomy.

In this analysis we did not attempt to differentiate intrapulmonary metastases from second primary lung cancers. However, there was no difference in survival between patients with multiple tumors of the same histology (which would include patients with intrapulmonary metastases) and patients with tumors of different histology. Similarly, there was no difference in survival among patients who under- 


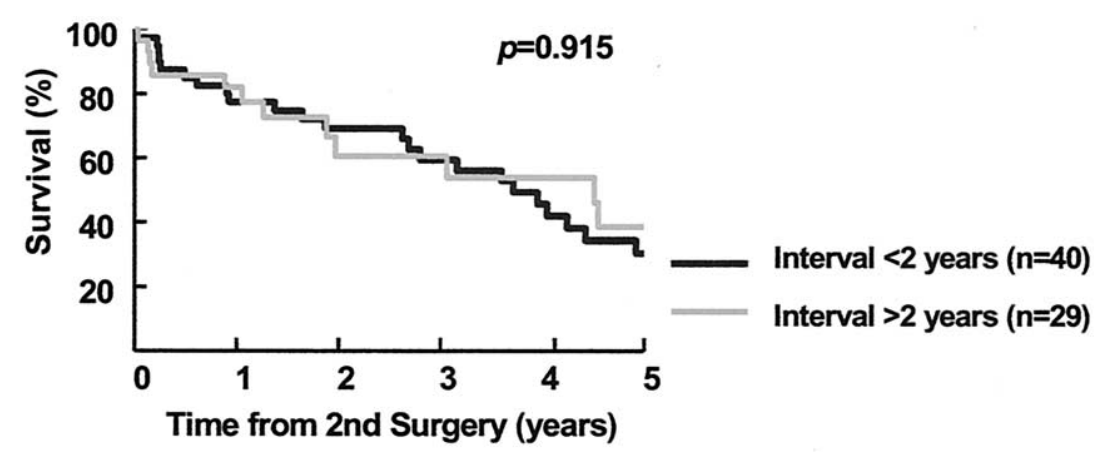

Figure 4. Five-year actuarial survival from the time of the second resection of the entire group stratified by the interval between resections.

went resection of a metachronous lesion within 2 years and those patients whose interval between resections was greater than 2 years. Although molecular techniques have been developed to compare tumors of the same histology in an attempt to differentiate intrapulmonary metastases from metachronous lung cancers, ${ }^{24,25}$ these techniques were not used in this series and are of no value in the preresection analysis of tumor cytology.

All of these patients were carefully staged by means of CT imaging of the chest and upper abdomen to include the liver and adrenal glands. In addition, extrathoracic metastases were excluded by using CT or magnetic resonance imaging of the brain and nuclear bone scans. More recently, positron emission tomography imaging has been used to exclude distant metastatic spread.

The management of patients in whom a second primary lung cancer develops remains a challenging problem. However, there is a small but real group of patients who appear to benefit from surgical resection of their second NSCLC.

Therefore we believe that the algorithm used to evaluate these patients for surgical resection of the second lesion should be identical to that used at the time of the first resection, and we continue to recommend complete surgical resection of metachronous NSCLC in patients with adequate physiologic reserve.

\section{References}

1. Mountain CF. Revisions in the International System for Staging Lung Cancer. Chest. 1997;111:1710-7.

2. Jemal A, Murray T, Samuels A, Ghafoor A, Ward E, Thun MJ. Cancer statistics, 2003. CA Cancer J Clin. 2003;53:5-26.

3. Martini N, Burt ME, Bains MS, McCormack PM, Rusch VW, Ginsberg RJ. Survival after resection of stage II non-small cell lung cancer. Ann Thorac Surg. 1992;54:460-5.

4. Ginsberg RJ, Rubinstein LV. Randomized trial of lobectomy versus limited resection for T1 N0 non-small cell lung cancer. Lung Cancer Study Group. Ann Thorac Surg. 1995;60:615-22.

5. Martini N, Bains MS, Burt ME, et al. Incidence of local recurrence and second primary tumors in resected stage I lung cancer. $J$ Thorac Cardiovasc Surg. 1995;109:120-9.

6. Battafarano RJ, Piccirillo JF, Meyers BF, et al. Impact of comorbidity on survival after surgical resection in patients with stage I non-small cell lung cancer. $J$ Thorac Cardiovasc Surg. 2002;123:280-7.

7. Martini N, Bains MS, Burt ME, et al. Incidence of local recurrence and second primary tumors in resected stage I lung cancer. J Thorac Cardiovasc Surg. 1995;109:120-9.

8. Johnson BE, Cortazar P, Chute JP. Second lung cancers in patients successfully treated for lung cancer. Semin Oncol. 1997;24:492-9.

9. Johnson BE. Second lung cancers in patients after treatment for an initial lung cancer. J Natl Cancer Inst. 1998;90:1335-45.

10. Walsh GL, O'Connor M, Willis KM, et al. Is follow-up of lung cancer patients after resection medically indicated and cost-effective? Ann Thorac Surg. 1995;60:1563-70.

11. Virgo KS, Johnson FE, Naunheim KS. Follow-up of patients with thoracic malignancies. Surg Oncol Clin N Am. 1999;8:355-69.

12. Gilbert S, Reid KR, Lam MY, Petsikas D. Who should follow up lung cancer patients after operation? Ann Thorac Surg. 2000;69:1696-700.

13. Westeel V, Choma D, Clement F, et al. Relevance of an intensive postoperative follow-up after surgery for non- small cell lung cancer. Ann Thorac Surg. 2000;70:1185-90.

14. Colice GL, Rubins J, Unger M. Follow-up and surveillance of the lung cancer patient following curative-intent therapy. Chest. 2003; 123(suppl):272S-83S.

15. Watanabe Y, Shimizu J, Oda M, Tatsuzawa Y, Hayashi Y, Iwa T. Second surgical intervention for recurrent and second primary bronchogenic carcinomas. Scand J Thorac Cardiovasc Surg. 1992; 26:73-8

16. Faber LP. Resection for second and third primary lung cancer. Semin Surg Oncol. 1993;9:135-41.

17. Adebonojo SA, Moritz DM, Danby CA. The results of modern surgical therapy for multiple primary lung cancers. Chest. 1997;112:693701.

18. Okada M, Tsubota N, Yoshimura M, Miyamoto Y. Operative approach for multiple primary lung carcinomas. $J$ Thorac Cardiovasc Surg. 1998;115:836-40.

19. Asaph JW, Keppel JF, Handy JR Jr, Douville EC, Tsen AC, Ott GY. Surgery for second lung cancers. Chest. 2000;118:1621-5.

20. van Rens MT, Zanen P, de la Riviere AB, Elbers HR, van Swieten HA, van den Bosch JM. Survival after resection of metachronous non-small cell lung cancer in 127 patients. Ann Thorac Surg. 2001;71:309-13.

21. Doddoli C, Thomas P, Ghez O, Giudicelli R, Fuentes P. Surgical management of metachronous bronchial carcinoma. Eur J Cardiothorac Surg. 2001;19:899-903.

22. Aziz TM, Saad RA, Glasser J, Jilaihawi AN, Prakash D. The management of second primary lung cancers. A single centre experience in 15 years. Eur J Cardiothorac Surg. 2002;21:527-33.

23. Martini N, Melamed MR. Multiple primary lung cancers. J Thorac Cardiovasc Surg. 1975;70:606-12.

24. Huang J, Behrens C, Wistuba I, Gazdar AF, Jagirdar J. Molecular 
analysis of synchronous and metachronous tumors of the lung: impact on management and prognosis. Ann Diagn Pathol. 2001;5:321-9.

25. Hiroshima K, Toyozaki T, Kohno H, Ohwada H, Fujisawa T. Synchronous and metachronous lung carcinomas: molecular evidence for multicentricity. Pathol Int. 1998;48:869-76.

\section{Discussion}

Dr Richard I. Whyte (Stanford, Calif). First, I would like to congratulate Dr Battafarano on a very interesting article and one that certainly adds to our body of knowledge about metachronous lung cancers.

I have 2 comments and 3 questions. My first comment gets to the purpose of the study, which you described here as to determine the effect of surgical intervention on metachronous lung cancers. In your article, however, I think you described the purpose as being to determine the effect of completely resected metachronous lung tumors on survival. Therefore in one sense it is comparing whether surgical intervention makes a difference, which is hard to answer if you have no patients who did not have an operation, whereas you can compare the survival of those who had metachronous tumors with those who did not in your current study.

The other question gets to the conclusion that this should be done on all patients with metachronous tumors, and what I noticed in reviewing the data is that the patients who had stage I disease from their primary tumor had a 5-year survival of $75 \%$ versus down to $42 \%$ in the metachronous patients. Therefore it is substantially lower, perhaps not significantly, but I think substantially lower, whereas in those patients who had more advanced-stage disease, the 5-year survival was only $10 \%$. And you had a $6 \%$ mortality. I am not sure that your $10 \%$ survival even accounts for that $6 \%$ mortality. Therefore those patients do what appears to be extremely poorly, and it certainly at least makes one address the issue that these patients should be very thoroughly staged before offering them an operation. My questions are 3-fold. First, given a $1 \%$ to $2 \%$ incidence of new cancers, you are reporting on $3 \%$ of your 2100 patients over the time period, and my question is what happened to the rest of the cancers?

Dr Battafarano. This is a selected group of patients, and even in the patients who had more advanced disease, most of this was found at the time of the operation. As you know, our group routinely uses mediastinoscopy, and therefore any patients with a positive mediastinoscopy-biopsy result would be excluded. That is why there is such a propensity for patients with stage I disease in this group. But what happened to the other patients? Many of those patients were excluded because at the time of our staging evaluation, they were found to have more advanced disease or such advanced disease that they would not tolerate the pulmonary resection necessary to enjoy complete resection on the basis of their pulmonary function. For instance, they would need a completion pneumonectomy, and their pulmonary function would not allow. I do not have the exact denominator. However, the percentage of our patients who underwent a second resection was about $3 \%$ of the total volume of patients who underwent pulmonary resection for lung cancer during that period. Others who have reported on their metachronous experience have proportions that have been more in the $4 \%$ range, and one article had results as high as $5 \%$. Therefore I think our selection might have been even a little bit more rigorous or we had a patient population whose pulmonary function would not have allowed complete resection of their disease at the time of metachronous resection.

Dr Whyte. On the basis of $1 \%$ to $2 \%$ per year, you would think in that follow-up period that you would get more than a total of $3 \%$ of your overall cases. That kind of gets to the second question. Where were these patients followed after their original operation? Were they followed by the division of cardiothoracic surgery, the section of thoracic surgery, or were they sent elsewhere and to come back if they had a new primary? And what happened in the interim? Were they followed with chest radiography or CT scans? How often? What was the frequency of that type of follow-up? That will help determine how many new patients with cancer you see.

Dr Battafarano. We follow all of our patients postoperatively. They are seen every 4 months for the first 3 years and then every 6 months thereafter. They are seen initially with chest radiography. If they have any abnormality on their chest X-ray films, we will perform a CT scan. Therefore we do follow them closely, and those patients in this study were identified by us in our normal surveillance program. But you are right, it is a small but real group that we identify who are able to withstand a second resection.

Dr Whyte. The last question concerns the fact that the pulmonary function test results were fairly similar, and you did not, in your article, say whether they were statistically significantly different. There was some slight decrease in forced expiratory volume in 1 second in the persons undergoing a second operation, but they were not all that different, yet $42 \%$, or nearly half, had wedge resections. I think most of us think that is not the best operation for lung cancer. I was wondering how you decided which patients would get lobectomies and which patients would get wedge resections.

Dr Battafarano. Well, I think it is individual. As you know, with every retrospective series, you cannot identify each of those things. I think the first thing is that all of the people in this series had negative margins on their permanent section. Therefore I think that if it was the surgeon's decision that they could perform a wide wedge excision on the lesion and wanted to preserve function that was a decision made by the surgeon. You can see from the series, though, that 29 underwent wedges, but most of them still underwent lobectomy or more. Therefore the idea of taking those patients to the operating room is to get a complete resection. But I think for the smaller lesions, the T1 lesions that were peripheral, that was the individual surgeon's decision to make on the basis of their pulmonary function tests and the location of the lesions whether a wedge resection was performed.

Dr Whyte. But given your choice, you prefer a lobe, I take it?

Dr Battafarano. Yes, for sure. We are very aggressive in performing anatomic resections on all NSCLCs.

Dr Scott J. Swanson (New York, NY). You mentioned mediastinoscopy a minute ago. Can you tell me what percentage of your patients initially had mediastinoscopy, and then how you did your preresectional mediastinal evaluation the second time around?

Dr Battafarano. Well, it varied partly because of the time sequence. If a person had a mediastinoscopy performed previously, then unless there were target lymph nodes or something, then a mediastinoscopy was not routinely done. In the first group of patients, a mediastinoscopy was performed in all 69 patients before their first resection. Again, there might have been persons in 
the series who had a large target lesion and not in the series of 69 but during the process of following our patients. If they had a target lesion on CT or other imaging, we will perform repeat mediastinoscopy.

Dr Swanson. How many had a repeat mediastinoscopy?

Dr Battafarano. I think it was only 15.

Dr Swanson. And if mediastinal sampling is important the first time around and you performed it for all patients, is it not equally important the second time around, and should you not do some evaluation, endoscopic ultrasonography with fine-needle aspiration, or thoracoscopic sampling to get at that same information?

Dr Battafarano. Well, on the basis of the series there, and you can see it by the second resection, it was very much bent toward stage I lesions. What happened was that all those patients then had mediastinal lymph nodes biopsied at the time of the operation.

Dr Swanson. Through a thoracotomy?

Dr Battafarano. Exactly.
Dr Daniel L. Miller (Atlanta, Ga). Did you look to see whether there was any difference in how the patient presented for a second resection, if it was found on incidental screening within your follow-up clinic or if it was in regard to symptoms? I think that is very important because when we looked at our resection after pneumonectomy while at the Mayo Clinic, we found that it made a tremendous difference in survival if they presented with symptoms compared with when they are found incidentally on screening.

Dr Battafarano. The large majority of these patients were asymptomatic at the time of diagnosis. But again, being retrospective, I think now many more of our more recent patients are getting CTs, either by us in the follow-up clinic because of an abnormal chest X-ray film or they will come from an outside physician with a CT, possibly even performed for another reason if they have had another malignancy. But the large majority of these patients were asymptomatic. 